Annals of Pure and Applied Mathematics

Vol. 15, No. 2, 2017, 271-276

ISSN: 2279-087X (P), 2279-0888(online)

Published on 11 December 2017

www.researchmathsci.org

DOI: http://dx.doi.org/10.22457/apam.v15n2a13

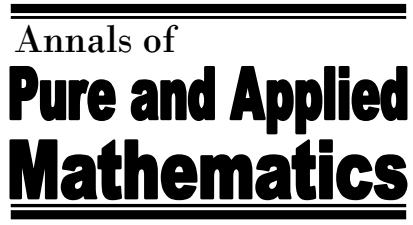

\title{
Analysis of Priority Queuing Models: L - R Method
}

\section{W. Ritha ${ }^{1}$ and S.Josephine Vinnarasi ${ }^{2}$}

Department of Mathematics

Holy Cross College (Autonomous)

Tiruchirappalli - 2, Tamil Nadu, India

Email: ritha_prakash@yahoo.co.in

Received 18 November 2017; accepted 7 December 2017

Abstract. Priority queuing models have a wide range of application in computer network system. In this paper the performance measures of fuzzy priority queuing model are computed using L- R method. L - $\mathrm{R}$ method is convenient and flexible compared to other methods. Numerical illustration is given to check the validity of the proposed method.

Keywords: Fuzzy queue, priority discipline performance measure, L-R method and triangular fuzzy numbers.

AMS Mathematics Subject Classification (2010): $03 \mathrm{E} 72$

\section{Introduction}

Queuing models play an indispensable role for capacity planning in practical situations. In today's networking environment, queuing models are basically relevant to service organizations and suggest ways and means to improve the efficiency of the service. A queue provides service at different alternatives on the basis of first-in-first out, last come first served, random selection and priority selection.

An efficient priority scheme is of great importance in the design and construction of telecommunication networks. We discuss and analyze the priority discipline fuzzy queuing model in two cases: no priority discipline, preemption priority by L- R Method. In a preemptive priority queue, an element with higher priority is served before an element with lower priority is already present in the service when the higher element arrives to the system. The order of the entries in the queue may change according to the priority scheme.

In fuzzy logic literature, fuzzy queues are extensively studied by researchers like $\mathrm{Li}$ and Lee [1], Buckley [16], Negi and Lee [2], Kao et al. [8], Chen [8] have analyzed fuzzy queues using Zadeh's extension principle, Ritha and Robert [15] analyzed fuzzy queues using DSW Algorithm, Ritha and Menon [11]. In this paper, L-R fuzzy number, arithmetic of L-R fuzzy numbers and triangular fuzzy number concepts are utilized for analysis of priority queuing models.

\section{Preliminaries}

L-R fuzzy number: A fuzzy number $\widetilde{M}$ is said L-R fuzzy number if only if there exists three real numbers $\mathrm{m}, \mathrm{a}>0, \mathrm{~b}>\mathrm{a}$ and two positive, continuous and decreasing functions $\mathrm{L}$ and $\mathrm{R}$, from $\mathrm{R}$ to $[0,1]$, such that 
W.Ritha and S.Josephine Vinnarasi

$$
\begin{gathered}
\mathrm{L}(0)=\mathrm{R}(0)=1 \\
\mathrm{~L}(1)=0, \mathrm{~L}(\mathrm{x})>0 \\
\mathrm{R}(1)=0, \mathrm{R}(\mathrm{x})>0, \\
\eta_{\widetilde{M}}(x)=\left\{\begin{array}{cc}
L\left(\frac{m-x}{a}\right) & \text { if } x \in[m-a, m] \\
R\left(\frac{x-m}{b}\right) & \text { if } x \in[m, m+b] \\
0 & \text { otherwise }
\end{array}\right.
\end{gathered}
$$

The L-R representation of the fuzzy number $\widetilde{M}$ is $\widetilde{M}=\langle\mathrm{m}, \mathrm{ab}\rangle_{\mathrm{LR}}$ where $\mathrm{m}$ is called the mean/ mode/ modal value of $\widetilde{M}$, a and $\mathrm{b}$ are the left spread and right spread of $\widetilde{M}$.

\section{Arithmetic of L-R fuzzy numbers:}

Suppose there are two L-R fuzzy numbers of the same type $\widetilde{M}=\langle\mathrm{m}, \mathrm{a}, \mathrm{b}\rangle_{\mathrm{LR}}$ and $\widetilde{N}=\langle\mathrm{n}, \mathrm{c}, \mathrm{d}\rangle_{\mathrm{LR}}$ then

1. $\widetilde{M}+\widetilde{N}=\langle\mathrm{m}+\mathrm{n}, \mathrm{a}+\mathrm{c}, \mathrm{b}+\mathrm{d}\rangle$

2. $\tilde{M}-\widetilde{N}=\langle\mathrm{m}-\mathrm{n}, \mathrm{a}+\mathrm{d}, \mathrm{b}+\mathrm{c}\rangle_{\mathrm{LR}}$

3. $\tilde{M} \cdot \widetilde{N}=\langle\mathrm{mn}, \mathrm{mc}+\mathrm{na}-\mathrm{ac}, \mathrm{md}+\mathrm{nb}+\mathrm{bd}\rangle$

4. $\frac{\widetilde{M}}{\widetilde{N}}=\frac{\langle m, a, b\rangle_{L R}}{\langle n, c, d\rangle_{L R}}=\left\langle\frac{m}{n}, \frac{m d}{n(n+d)}+\frac{a}{n}-\frac{a d}{n(n+d)}, \frac{m c}{n(n-c)}+\frac{b}{n}+\frac{b c}{n(n-c)}\right\rangle_{L R}$

\section{Triangular fuzzy numbers:}

A fuzzy number $\tilde{A}$ is said to be triangular fuzzy number if and only if there exists three real numbers $\mathrm{a}<\mathrm{b}<\mathrm{c}$ such that:

$$
\eta_{\widetilde{M}}(x)=\left\{\begin{array}{lc}
\left(\frac{x-a}{b-a}\right) & \text { if } a \leq x \leq b \\
\left(\frac{c-x}{c-b}\right) & \text { if } b<x \leq c \\
0 & \text { otherwise }
\end{array}\right.
$$

Denoted by $\tilde{A}=(\mathrm{a} / \mathrm{b} / \mathrm{c})$ or $\tilde{A}=(\mathrm{a}, \mathrm{b}, \mathrm{c})$

Remark: A triangular fuzzy number $\tilde{A}=(\mathrm{a} / \mathrm{b} / \mathrm{c})$ is always an L-R fuzzy number.

In L-R representation,

$\tilde{A}=(\mathrm{a} / \mathrm{b} / \mathrm{c})=\langle\mathrm{b}, \mathrm{b}-\mathrm{a}, \mathrm{c}-\mathrm{b}\rangle_{\mathrm{LR}} \quad$ for $\mathrm{L}(\mathrm{x})=\mathrm{R}(\mathrm{x})=\max (0,1-\mathrm{x})$

The following notations are used:

$\tilde{\lambda}$ : Fuzzy rate of arrival

$\tilde{\mu}$ : Fuzzy rate of service

$\tilde{C}$ : Average total cost of inactivity when there is no priority discipline 
Analysis of Priority Queuing Models: L - R Method

$\widetilde{C}^{\prime}$ : Average total cost of inactivity when there is preemption priority

\section{Fuzzy priority queues:}

Fuzzy priority queues are based on the concept of fuzzy set theory. Consider a priority queuing system with single server, infinite calling population in which arrival rate is $\tilde{\lambda}$ and service rate is $\tilde{\mu}$

We establish the priority discipline fuzzy queuing model using L-R technique for two cases:

(i) No priority discipline

(ii) Preemption priority discipline

(a) No priority queuing model:

Average total cost of inactivity when there is no priority discipline $\tilde{C}=\left(\begin{array}{lll}\tilde{C}_{1} & \tilde{\lambda}_{1}+\tilde{C}_{2} & \tilde{\lambda}_{2}\end{array}\right) \widetilde{W}$ with $\widetilde{W}=\frac{1}{\widetilde{\mu}-\tilde{\lambda}}$

(b) Preemption priority queuing model:

Average total cost of inactivity when there is preemption priority

$\tilde{C}^{\prime}=\tilde{C}_{1} \tilde{\lambda}_{1} \widetilde{W}_{1}+\tilde{C}_{2} \tilde{\lambda}_{2} \widetilde{W}_{2} \mathrm{~W}_{\mathrm{q}, \mathrm{i}}=\frac{\lambda}{\mu^{2}} \frac{1}{\left(1-\sigma_{i)\left(1-\sigma_{\mathrm{i}+1}\right)}\right.}$

where $\sigma_{1}=\frac{\lambda 1}{\mu}$, where $\sigma_{2}=\frac{\lambda 2}{\mu} \quad$ and $\sigma_{3}=0$

\section{L-R method description:}

Let the arrival rate and service rate be triangular fuzzy numbers such that

$\tilde{\lambda}=\left(\lambda_{1} / \lambda_{2} / \lambda_{3}\right)$ and $\tilde{\mu}=\left(\mu_{1} / \mu_{2} / \mu_{3}\right)$

$\widetilde{C}_{1}=\left(\mathrm{C}_{11} / \mathrm{C}_{12} / \mathrm{C}_{13}=\left\langle\mathrm{C}_{12}, \mathrm{C}_{12}-\mathrm{C}_{11}, \mathrm{C}_{13}-\mathrm{C}_{12}\right\rangle_{\mathrm{LR}}\right.$

$\tilde{C}_{2}=\left(\mathrm{C}_{21} / \mathrm{C}_{22} / \mathrm{C}_{23}=\left\langle\mathrm{C}_{22}, \mathrm{C}_{22}-\mathrm{C}_{21}, \mathrm{C}_{23}-\mathrm{C}_{22}\right\rangle_{\mathrm{LR}}\right.$

$\tilde{\lambda}_{1}=\left(\lambda_{11} / \lambda_{12} / \lambda_{13}\right)=\left\langle\lambda_{12}, \lambda_{12}-\lambda_{11}, \lambda_{13}-\lambda_{12}\right\rangle_{\mathrm{LR}}$

$\tilde{\lambda}_{2}=\left(\lambda_{21} / \lambda_{22} / \lambda_{23}\right)=\left\langle\lambda_{22}, \lambda_{22}-\lambda_{21}, \lambda_{23}-\lambda_{22}\right\rangle_{\text {LR }}$

$\tilde{\lambda}=\left(\lambda_{1} / \lambda_{2} / \lambda_{3}\right)=\left\langle\lambda_{2}, \lambda_{2}-\lambda_{1}, \lambda_{3}-\lambda_{2}\right\rangle_{\mathrm{LR}}$

$\tilde{\mu}=\left(\mu_{1} / \mu_{2} / \mu_{3}\right)=\left\langle\mu_{2}, \mu_{2}-\mu_{1}, \mu_{3}-\mu_{2}\right\rangle_{\text {LR }}$

The cost of inactivity when there is no priority discipline is given as:

$$
\tilde{c}=\frac{\widetilde{C_{1}} \widetilde{\lambda_{1}}+\widetilde{C_{2}} \widetilde{\lambda_{2}}}{\tilde{\mu}-\tilde{\lambda}}
$$


W.Ritha and S.Josephine Vinnarasi

$$
\begin{aligned}
& <C_{12}, C_{12}-C_{21}, C_{13}-C_{12}>\underset{L R}{C_{21}}-C_{23}, C_{22}, \lambda_{L R}-\lambda_{22}^{12}, \lambda_{22}^{12}-\lambda_{21}^{11}, \lambda_{32}^{13}-\lambda_{22}^{12}>{ }_{L R}{ }_{L R+<} C_{22}, \\
& <\mu_{2}, \mu_{2}-\mu_{1}, \mu_{3}-\mu_{2}>_{\text {LR }}-\lambda_{2}, \lambda_{2}-\lambda_{1}, \lambda_{3}-\lambda_{2}>_{\text {LR }} \\
& \frac{c_{12} \lambda_{12}+c_{22} \lambda_{22}}{\mu_{2}-\lambda_{2}}\left\{\begin{array}{c}
\frac{\left[c_{12} \lambda_{12}+c_{22} \lambda_{22}\right]\left[\mu_{3}-\mu_{2}+\lambda_{2}-\lambda_{1}\right]}{\mu_{2}-\lambda_{2)(} \mu_{3}-\lambda_{1} \lambda_{12}}+\frac{c_{12} \lambda_{12}-c_{11} \lambda_{11}+c_{22} \lambda_{22}-c_{21} \lambda_{21}}{\mu_{12}-\lambda_{2}+c_{22} \lambda_{22}-c_{21} \lambda_{21}} \\
\frac{c_{12} \lambda_{12}-\lambda_{2)(} \mu_{3}-\lambda_{1)}}{\mu_{2}}
\end{array}\right\}, \\
& \left\{\frac{\left[C_{12} \lambda_{12}+C_{22} \lambda_{22}\right]\left[\mu_{2}-\mu_{1}+\lambda_{3}-\lambda_{2}\right]}{\left(\mu_{2}-\lambda_{2}() \mu_{1}-\lambda_{3)}\right.}+\frac{C_{13} \lambda_{13}-C_{12} \lambda_{12}+C_{23} \lambda_{23}-C_{22} \lambda}{\mu_{2}{ }^{22} \lambda_{2}}\right. \\
& \left.+\frac{\left[C_{13} \lambda_{13}-C_{12} \lambda_{12}+C_{23} \lambda_{23}-C_{22} \lambda_{22}\right]\left[\mu_{2}-\mu_{1}+\lambda_{3}-\lambda_{2}\right]}{\mu_{2}-\lambda_{2)(} \mu_{1} \lambda_{3)}}\right\}
\end{aligned}
$$

The lower and upper bound of $\widetilde{N}$ are $N_{1}-N_{2}$ and $N_{1}+N_{3}$. Noting $\mathrm{m}=\mathrm{N}, \mathrm{N}=N_{1}-N_{2}$,

$\mathrm{V}=N_{1}+N_{3}$

The cost of inactivity for Preemptive priority queuing model is given as:

$$
\tilde{C}^{\prime}=\tilde{C}_{1} \tilde{\lambda}_{1} \widetilde{W}_{1}+\widetilde{C}_{2} \tilde{\lambda}_{2} \widetilde{W}_{2}
$$

where $\widetilde{W_{1}}=\left(\mathrm{W}_{11} / \mathrm{W}_{12} / \mathrm{W}_{13}=\left\langle\mathrm{W}_{12}, \mathrm{~W}_{12}-\mathrm{W}_{11}, \mathrm{~W}_{13}-\mathrm{W}_{12}\right\rangle_{\mathrm{LR}}\right.$ and

$\widetilde{W}_{2}=\left(\mathrm{W}_{21} / \mathrm{W}_{22} / \mathrm{W}_{23}\right)=\left\langle\mathrm{W}_{22}, \mathrm{~W}_{22}-\mathrm{W}_{21}, \mathrm{~W}_{23}-\mathrm{W}_{22}\right\rangle_{\mathrm{LR}}$

$\tilde{C}=\left\langle\mathrm{C}_{12} \lambda_{12}, \mathrm{C}_{12} \lambda_{12}-\mathrm{C}_{11} \lambda_{11}, \mathrm{C}_{13} \lambda_{13}-\mathrm{C}_{12} \lambda_{12}\right\rangle_{\mathrm{LR}}\left\langle\mathrm{W}_{12}, \mathrm{~W}_{12}-\mathrm{W}_{11}, \mathrm{~W}_{13}-\mathrm{W}_{12}\right\rangle_{\mathrm{LR}}+$

$<\mathrm{C}_{22} \lambda_{22}, \mathrm{C}_{22} \lambda_{22}-\mathrm{C}_{21} \lambda_{21}, \mathrm{C}_{23} \lambda_{23}-\mathrm{C}_{22} \lambda_{22}>_{\mathrm{LR}}<\mathrm{W}_{22}, \mathrm{~W}_{22}-\mathrm{W}_{21}, \mathrm{~W}_{23}-\mathrm{W}_{22}>_{\mathrm{LR}}$

$\tilde{C}=\left\langle\mathrm{C}_{12} \lambda_{12} \mathrm{~W}_{12}+\mathrm{C}_{22} \lambda_{22} \mathrm{~W}_{22}, \mathrm{C}_{12} \lambda_{12} \mathrm{~W}_{12}-\mathrm{C}_{11} \lambda_{11} \mathrm{~W}_{11}+\mathrm{C}_{22} \lambda_{22} \mathrm{~W}_{22}-\mathrm{C}_{21} \lambda_{21} \mathrm{~W}_{21}\right.$,

$\mathrm{C}_{13} \lambda_{13} \mathrm{~W}_{13}-\mathrm{C}_{12} \lambda_{12} \mathrm{~W}_{12+} \mathrm{C}_{23} \lambda_{23} \mathrm{~W}_{23}-\mathrm{C}_{22} \lambda_{22} \mathrm{~W}_{22}>_{\mathrm{LR}}$

\section{Let us illustrate the above model with an example:}

No priority discipline fuzzy queuing model are given with their fuzzy arrival rates and service rates with their costs

$\tilde{\lambda}=(44 / 45 / 46)=\langle 45,1,1\rangle_{\mathrm{LR}}$ and $\tilde{\mu}=(47 / 48 / 49)=\langle 48,1,1\rangle_{\mathrm{LR}}$

$\widetilde{\lambda_{1}}=(34 / 35 / 36)=\langle 35,1,1\rangle_{\mathrm{LR}}$ and $\widetilde{\lambda_{2}}=(32 / 33 / 34)=\langle 33,1,1\rangle_{\mathrm{LR}}$

$\widetilde{C_{1}}=(41 / 42 / 43)=\langle 42,1,1\rangle_{\mathrm{LR}}$ and $\widetilde{C_{2}}=(37 / 38 / 39)=\langle 38,1,1\rangle_{\mathrm{LR}}$ 
Analysis of Priority Queuing Models: L - R Method

The cost of inactivity is given as

$\widetilde{C}=\langle 908,392,1966\rangle_{\mathrm{LR}}$

On simplification,

Modal value $=908$, Left spread $=392$, Right spread $=1966$

From the above calculation, we consider the values for $\tilde{\lambda}, \tilde{\mu}, \widetilde{\lambda_{1}}, \widetilde{\lambda_{2}}, \widetilde{C_{1}}, \widetilde{C_{2}}$ with

$\widetilde{W_{1}}=(44 / 45 / 46)=\langle 45,1,1\rangle_{\mathrm{LR}} \quad$ and $\quad \widetilde{W}_{2}=(47 / 48 / 49)=\langle 48,1,1\rangle_{\mathrm{LR}}$

The cost of inactivity for non-preemptive priority fuzzy queue model is obtained as

$\widetilde{C^{\prime}}=\langle 126342,9358,9840\rangle_{\mathrm{LR}}$

\section{Conclusion}

In this paper, we have analyzed fuzzy priority queues by L-R Method, based on L-R fuzzy numbers, L-R fuzzy arithmetic using the concept of triangular fuzzy numbers. Fuzzy concept gives more appropriate result when compared with crisp results. L-R Method is short, flexible, convenient and approximate method for best explanatory results. L-R Method helps in further research work for evaluation of fuzzy queuing operating system.

\section{REFERENCES}

1. R.J.Li and E.S.Lee, Analysis of fuzzy queues, Computers and Mathematics with Applications, 17(7) (1989) 1143 - 1147.

2. D.S. Negi and E.S. Lee, Analysis and simulation of fuzzy queues, Fuzzy Sets and systems 46(1992), $321-330$

3. D.Dubois, H.Parade, Fuzzy Sets and Systems: Theory and Applications, Academic Press, New York, (1980)

4. M.Hans, Applied Fuzzy Arithmetic, An Introduction with Engineering Applications Springer - Verlag, Berlin Heidelberg, (2005)

5. W.Dong and H.C.Shah, Vertex method for computing functions on fuzzy variables, Fuzzy Sets and Systems, 24 (1987) 65-78

6. S.Drekic and D.G.Woolford, A preemptive priority queue with balking, European Journal of Operational Research, 164 (2) (2005) 387-401.

7. R.Groenevelt and E. Altman, Analysis of alternating - priority queuing models with (cross) correlated switchover times, Queuing Systems, 51(3-4) (2005) 199-247

8. C.Kao, C.C.Li and S.P.Chen, Parametric programming to the analysis of fuzzy queues, Fuzzy Sets and Systems, 107 (1999) 93-100.

9. J. P.Mukeba, R.Mabela and B. Ulungu, Sur la multiplication des nombres flousde type 1-r, Annals of Faculty of Sciences, University of Kinshasa,1(2014)33- 40

10. W.Ritha and Lilly Robert, Application of fuzzy set theory to retrial queues, International Journal of Algorithms, Computing and Mathematics, 2 (4) (2009) 9-18.

11. W.Ritha and S.B. Menon, Fuzzy n policy queues with infinite capacity, Journal of Physical Sciences, 15 (2011) 73-82.

12. R.Srinivasan, Fuzzy queuing model using DSW algorithm, International Journal of Advanced Research in Mathematics and Computer Applications, 1 (1) (2013) 1-6.

13. T.-Y.Wang, D.Y.Yang and M.-J.Li, Fuzzy analysis for the n-policy queues within finite capacity, International Journal of Information and Management Sciences, 21 (2010) 41-45.

14. H.J. Zimmermann, Fuzzy set theory and its applications, Springer Science + Business Media, New-York, fourth edition, (2001). 
W.Ritha and S.Josephine Vinnarasi

15. W.Ritha and Lilly Robert, Fuzzy queues with priority discipline, Applied Mathematical Sciences, 4 (2010) 575-582.

16. J.P. Mukeba Kanyinda, R. Mabela Makengo Matendo, B. Ulungu Ekunda Lukata, computing fuzzy queuing performance measures by L-R method, Journal of Fuzzy Set Valued Analysis, 1 (2015) 57-67.

17. J.J. Buckley, Elementary queuing theory based on possibility theory, Fuzzy Sets and Systems, 37 (1990) 43-52. 